\title{
Estresse ocupacional: causas e consequências
}

\author{
Occupational stress: causes and consequences
}

\author{
Claudia Eliza Papa do Prado ${ }^{1}$
}

\begin{abstract}
RESUMO | Contexto: O estresse é uma doença crônica recorrente que, em longo prazo, pode ocasionar incapacidade para o trabalho, gerando custos, perda de renda vitalícia e aposentadoria antecipada, além do risco de suicídio. Objetivos: Apresentar os fatores relacionados ao estresse ocupacional, ressaltando os mecanismos desencadeadores da doença, os principais sintomas e as medidas adotadas para melhorar a produtividade e a qualidade de vida do trabalhador. Métodos: Realizou-se um estudo descritivo com consulta às bases de dados nacionais LILACS e SciELO, e internacionais MEDLINE e PubMed, considerando-se o período de 1996 a 2015 e os critérios de inclusão e exclusão dos artigos. Resultados: O estresse ocupacional é um estado em que ocorre desgaste do organismo humano e/ou diminuição da capacidade de trabalho. Por si só, não é capaz de desencadear uma enfermidade orgânica ou provocar uma disfunção significativa na vida do indivíduo. Para que isso ocorra, é necessário que outras condições estejam presentes, como a vulnerabilidade orgânica ou a forma inadequada de avaliar e enfrentar a situação estressante. A preocupação científica com a questão do estresse ocupacional reside na sua provável relação com o adoecimento ou sofrimento que acarreta. Conclusão: Os principais agentes estressores podem ser os fatores ambientais e organizacionais, no entanto o diagnóstico é essencialmente clínico baseado nos sintomas da doença, que podem ser físicos, psicológicos e comportamentais. Para se intervir na saúde do trabalhador, torna-se imperiosa a combinação de abordagens e enfoques, transformações organizacionais do trabalho, mudanças urbanas e fatores de riscos industriais e ambientais.
\end{abstract}

Palavras-chave I esgotamento profissional; sinais e sintomas; saúde do trabalhador; qualidade de vida.

ABSTRACT | Background: Stress is a chronic recurrent disease that may lead to work inability in a long term, which generates costs, loss of lifetime income, early retirement, and increases the risk of suicide. Objectives: To reveal the factors associated with occupational stress, and emphasize disease triggers, main symptoms, and the actions implemented to improve productivity and worker's quality of life. Methods: A descriptive study was carried out based on a search in the national databases LILACS and SciELO, and in the internacional databases MEDLINE and PubMed, considering the period of 1996-2015 and the criteria for inclusion and exclusion of articles. Results: Occupational stress is a condition that causes mental and physical stress and reduces working ability. It alone does not cause an organic disease or a significant functional impairment. This consequences may occurs if other conditions are present, such as organic vulnerability or inability to assess and cope with the stressful situation. The concern of the scientific community with occupational stress is related to its possible association with illness or suffering. Conclusion: Stressors may be environmental and organizational factors; however, diagnosis is essentially clinical and based on disease symptoms that may be physical, psychological, and behavioral. A mix of approaches, organizational changes related to the work, urban changes, and industrial and environmental risk factors management are essential for intervention actions toward workers' health.

Keywords I burnout, professional; signs and symptoms; occupational health; quality of life. 


\section{INTRODUÇÃO}

Os trabalhadores sofrem impacto significativo com as mudanças sociais, econômicas e tecnológicas que atualmente a sociedade enfrenta, dando novos rumos e sentido ao trabalho. O profissional da era globalizada participa de um cenário constituído por diversos fatores, como a alta competitividade, a ascensão da mão de obra terceirizada e a concorrência acirrada, ocasionando desgastes fisiológicos e cognitivos no corpo humano. Os trabalhadores cujas atividades dependem de alto grau de responsabilidade, agilidade de decisão e outras vertentes que exijam resultados satisfatórios estão cada vez mais renunciando ao lazer e ao descanso que o corpo e a mente necessitam para se restabelecerem ${ }^{1}$.

No Brasil, assim como em outros polos industriais, a busca pela adaptação a esse novo panorama tem gerado impacto representativo na saúde dos trabalhadores. Nesse contexto, o estresse ocupacional pode ser entendido como um conjunto de perturbações psicológicas ou sofrimento psíquico associado às experiências de trabalho ${ }^{2,3}$. Em razão dessas repercussões negativas sobre o indivíduo, surgiram propostas que visam a adequar o trabalho e a estrutura das organizações às necessidades dos trabalhadores, buscando a satisfação destes e a melhoria de seu desempenho profissional ${ }^{4}$.

Mediante a relevância do tema, este artigo tem como objetivo apresentar os fatores relacionados ao estresse ocupacional, ressaltando os mecanismos desencadeadores da doença, os principais sintomas dela e as medidas adotadas para melhorar a produtividade e a qualidade de vida do trabalhador.

\section{MÉTODOS}

\section{ESTRATÉGIA DE BUSCA}

Realizou-se um estudo descritivo, por meio de revisão da literatura, com consulta às bases de dados nacionais LILACS e SciELO, e internacionais MEDLINE e PubMed, considerando-se o período de 1996 a 2015. Na seleção dos descritores, foi utilizada a terminologia em saúde consultada nos Descritores em Ciências da Saúde (DeCS) a partir das seguintes palavras-chaves/keywords: "esgotamento profissional" (Burnout profissional), "sinais e sintomas" (signs and symptoms), "saúde do trabalhador" (occupational health) e "qualidade de vida" (quality of life). Vale ressaltar que esses termos foram utilizados de forma conjunta e isolados inseridos, independentemente, nas bases de dados.

\section{SELEÇÃO DOS ARTIGOS}

\section{Critérios de inclusão}

Foram incluídos artigos de periódicos e fontes não indexadas (livros), publicados nos idiomas inglês e português.

\section{Critérios de exclusão}

Foram excluídos os artigos publicados em outros idiomas e que não foram obtidos na íntegra. De acordo com os critérios de inclusão e exclusão preestabelecidos, os estudos foram submetidos à análise dos títulos e resumos para que houvesse uma seleção inicial dos artigos. Posteriormente, os artigos selecionados foram acessados na íntegra e uma nova análise foi realizada, restando apenas artigos que se encaixassem exatamente nos critérios de inclusão estabelecidos inicialmente.

\section{RESULTADOS E DISCUSSÃO}

\section{CONCEITO, TIPOLOGIAS BÁSICAS E FASES DO ESTRESSE}

O conceito de estresse, originalmente utilizado na Física para descrever uma força ou um conjunto de forças que, aplicadas a um corpo, tendem a desgastá-lo ou deformá-lo, foi estudado por Selye ${ }^{5}$ considerando-se as reações desencadeadas pelo organismo exposto a diferentes situações nocivas à saúde. Os resultados mostraram que o estresse é caracterizado por uma síndrome específica de fatos biológicos, apresentando-se como uma resposta inespecífica do corpo diante de exigências às quais está sendo submetido, manifestando-se de forma positiva (eustresse), que motiva e provoca a resposta adequada aos estímulos estressores, ou negativa (distresse), que intimida o indivíduo diante de situação ameaçadora, com predominância de emoções de ansiedade, medo, tristeza e raiva ${ }^{6,7}$.

A manutenção do estado de homeostase é essencial para a vida, sendo constantemente desafiado por forças internas ou externas que atuam no psiquismo do indivíduo, ativando emoções e desencadeando uma reação dos sistemas nervoso e glandular que atinge, principalmente, o nível físisco ${ }^{8}$. Com o propósito de minimizar os efeitos danosos do estresse, o organismo ativa os sistemas sensoriais que desencadeiam uma 
resposta aguda, a fim de induzir uma rápida mobilização de energia aos locais apropriados?.

Conforme citado anteriormente, o estresse produz reações de defesa e adaptação diante do agente estressor, as quais são classificadas em fase de alarme, resistência e exaustão ${ }^{10}$. A fase de alarme inicia-se com os estímulos estressores que provocam resposta rápida do organismo (luta e fuga). Entretanto, esse estágio não se mantém por muito tempo, pois o esforço maior não visa somente à manutenção da harmonia interior mas ao enfrentamento da situação ameaçadora. As alterações observadas no organismo, nessa fase, incluem aumento das frequências cardíaca e respiratória, e da pressão arterial; contração do baço; liberação de glicose pelo fígado; redistribuição sanguínea e dilatação das pupilas.

$\mathrm{Na}$ fase de resistência, o indivíduo tenta se adaptar à nova situação com o propósito de restabelecer o equilíbrio interno, pois o organismo apresenta um desgaste maior, dificuldades de memória e está mais vulnerável a doenças. Os sintomas mais comumente observados são o tremor muscular, fadiga física, desânimo, irritabilidade, dificuldade de concentração e instabilidade emocional. Por fim, a fase de exaustão consiste em uma extinção da resistência em decorrência de falhas nos mecanismos de adaptação. É considerada a condição mais crítica relacionada ao estresse, pois, após exposições repetidas ao mesmo estressor, o organismo pode desenvolver doenças graves ou, até mesmo, entrar em colapso ${ }^{11}$. Em complementação, Lipp ${ }^{12}$ identificou outra fase do processo de estresse, denominada de quase-exaustão, caracterizada por um enfraquecimento do indivíduo que não está conseguindo adaptar-se ou resistir ao estressor. As afecções começam a surgir, porém não são tão graves quanto na fase de exaustão.

\section{ESTRESSE OCUPACIONAL}

O conjunto e a divisão de tarefas que compõem a carga de trabalho do profissional estão associados a importantes estressores laborais, os quais podem sofrer agravos significativos em razão de condições precárias de organização do trabalho, que vão desde a baixa valorização e remuneração, descompasso entre tarefas prescritas e realizadas, até a escassez severa de recursos e problemas de infraestrutura ${ }^{13}$.

De acordo com Genuíno et al. ${ }^{1}$, o estresse ocupacional refere-se aos estímulos do ambiente de trabalho que exigem resposta. A caracterização de um fenômeno de estresse depende da percepção do indivíduo em avaliar os eventos como estressores, portanto o cognitivo tem papel importante no processo que ocorre entre os estímulos potencialmente estressores e as respostas do indivíduo a eles. $\mathrm{O}$ termo estressor ocupacional designa estímulos que são gerados no trabalho e têm consequências físicas ou psicológicas negativas para um maior número de indivíduos expostos a eles. Consideram-se agentes estressores os fatores extraorganizacionais e organizacionais, individuais e de grupo.

Para a abordagem do estresse ocupacional, são consideradas as vertentes biológica, psicológica e sociológica, que, apesar de distintas, são complementares e estão interligadas. Na biológica, o estresse é caracterizado, essencialmente, pelo grau de desgaste do corpo. Os processos afetivos, emocionais e intelectuais do indivíduo correspondem à abordagem psicológica, ou seja, é a maneira pela qual este se relaciona com as outras pessoas e com o mundo ao seu redor. Em adição, a sociológica refere-se à compreensão das variáveis que se estabelecem no contexto da sociedade. $\mathrm{O}$ diagnóstico dos sinais e sintomas do estresse ocupacional é essencialmente clínico, baseado nos rastreamentos individual e do risco nas situações de trabalho ${ }^{8}$.

\section{SIINDROME DE BURNOUT}

Apontada como um fenômeno em expansão na atualidade, a síndrome de Burnout (SB), também conhecida como esgotamento profissional no Brasil, tem recebido diferentes denominações ${ }^{14}$. A definição mais amplamente aceita é baseada na perspectiva psicossocial que busca identificar as condições no ambiente de trabalho que conduzem ao Burnout e os sintomas específicos que caracterizam a síndrome ${ }^{15,16}$.É considerada como uma resposta prolongada a estressores emocionais e interpessoais crônicos no trabalho, sendo classificada como exaustão emocional, despersonalização e ineficácia ${ }^{17,18}$. Manifesta-se basicamente por sintomas de fadiga persistente, falta de energia, adoção de condutas de distanciamento afetivo, insensibilidade, indiferença ou irritabilidade relacionadas ao trabalho, além de sentimentos de ineficiência e baixa realização pessoal ${ }^{19}$.

De acordo com Maslach et al. ${ }^{18}$, o Burnout é desenvolvido por meio das fases de:

- idealismo: o trabalho é interessante e preenche as necessidades do indivíduo;

- realismo: as expectativas iniciais não foram supridas, o trabalho não satisfaz as necessidades, as recompensas e o reconhecimento são escassos;

- estagnação e frustação: o entusiasmo e a energia iniciais se transformam em fadiga crônica e irritabilidade; e

- apatia: a pessoa tem a sensação de desespero, fracasso e perda da autoestima e autoconfiança. 
O diagnóstico da síndrome é complexo, pois seus efeitos apresentam consequências variáveis em termos psicológicos, implicações físicas e alteração de conduta. No plano das implicações psíquicas, o indivíduo acometido descompensa-se, responde de modo inadequado à tensão e aos estímulos do ambiente de trabalho, perde o eixo, encontra dificuldade de aprendizagem, tem insônia, pesadelos, impotência e apatia, extingue laços afetivos e evita restabelecer novos vínculos, isola-se, afasta-se dos familiares, e demonstra desinteresse pelo emprego ${ }^{20}$. A diferença entre estresse e síndrome de Burnout reside no fato de que no estresse são observados pontos positivos e negativos, além da predominância de sintomas físicos com emoções exageradas. Em contrapartida, no Burnout, são marcantes apenas os aspectos negativos (distresse).

Existem alguns tipos de atividades cujos profissionais são mais propensos a desenvolverem o desgaste emocional. De acordo com Benevides-Pereira ${ }^{21}$, a síndrome de Burnout acomete, principalmente, profissionais que prestam assistência ou são responsáveis pelo desenvolvimento ou cuidado de outros. Essa síndrome foi reconhecida como risco ocupacional para profissões que envolvem cuidados com saúde, educação e serviços humanos ${ }^{22,23}$.

Fabichak et al. ${ }^{24}$ conduziram um estudo com médicos residentes, com o objetivo de identificar a frequência da SB e a exposição a estressores organizacionais presentes nas condições de trabalho. Os resultados mostraram que 50\% dos participantes apresentavam critérios para SB, sendo a maioria do gênero feminino, com média de idade de 26,6 anos. Os autores enfatizaram a importância de abordagem preventiva nas situações que exercem impacto negativo sobre a saúde dos profissionais expostos.

\section{PREVENÇÃO E ENFRENTAMENTO DO ESTRESSE OCUPACIONAL}

O estresse ocupacional afeta o indivíduo, a prestação de serviço e a qualidade dele, sendo necessário o trabalho preventivo. A prevenção é de fundamental importância porque enfatiza a dimensão humana e sinaliza os cuidados quanto ao respeito à saúde do trabalhador. O enfrentamento do estresse tem como objetivo principal minimizar ou moderar os efeitos sobre o bem-estar emocional e físico do indivíduo. Algumas estratégias aliviam temporariamente e podem ser mal adaptativas em longo prazo. Outras são ineficazes, pois o estressor não é eliminado e sua recorrência não pode ser impedida. Entretanto, estratégias com foco na emoção e no problema são mais efetivas, pois o indivíduo lida diretamente com o estressor, reduzindo as demandas deste.
A redução do estresse envolve vários aspectos, como alimentação, relaxamento, exercício físico, estabilidade emocional e qualidade de vida, e esses fatores devem ser considerados como um todo para que se possam alcançar resultados satisfatórios. As práticas gerenciais do novo ambiente de trabalho também colaboram para que o estresse não se torne excessivo, pois propiciam melhor comunicação, seleção adequada, concessão de poder e participação, metas definidas, capacitação de funcionários e apoio às famílias ${ }^{25}$.

\section{QUALIDADE DE VIDA NO TRABALHO}

A qualidade de vida no trabalho (QVT) tem recebido diferentes denominações, entretanto existe um consenso de que uma maior humanização, aumento do bem-estar do trabalhador e maior participação dele nas decisões e nos problemas da empresa relacionam-se diretamente com a qualidade de vida. A QVT envolve fatores intrínsecos e extrínsecos do cargo, afetando tanto as atitudes pessoais quanto as comportamentais, com relevância na produtividade individual e coletiva. Entende-se que motivação, adaptabilidade, criatividade e vontade de inovação ou de aceitar mudanças estão diretamente ligadas à QVT, tendo representativa influência na produtividade e lucratividade das organizações ${ }^{26}$.

Os fatores organizacionais que mais afetam a QVT são a sobrecarga no trabalho, falta de estímulos e de perspectivas, ruídos, alteração do sono, necessidade de mudanças e ergonomia. Nesse contexto, salienta-se a importância da implantação de programas de qualidade de vida no ambiente de trabalho. Um dos obstáculos para a implementação de tais programas é que alguns gestores os consideram um aumento nos custos, e não investimento intelectual e profissional. Torna-se imprescindível estimular o envolvimento e o comprometimento do trabalhador, e essa é uma ideia que deve ser praticada pelos gestores das organizações ${ }^{27}$. Envolver os indivíduos na estruturação de suas funções e fornecer a capacitação e as ferramentas necessárias para um desempenho eficiente é uma opção válida para a melhoria da qualidade e da produtividade no trabalho a ser desenvolvido.

\section{CONCLUSÕES}

O estresse ocupacional vem sendo considerado um problema generalizado afetando os trabalhadores, a organização e a sociedade como um todo. Este, por si só, não é 
capaz de desencadear uma enfermidade orgânica ou provocar uma disfunção significativa na vida do indivíduo. Para que isso ocorra, é necessário que outras condições estejam presentes, como a vulnerabilidade orgânica ou uma forma inadequada de avaliar e enfrentar a situação estressante.

As estratégias para o tratamento devem ser iniciadas quando se reconhece que as pressões na vida individual atingiram um ponto tal que estão causando problemas físicos, psicológicos e de comportamento. Acredita-se que para preservar a saúde mental e física, bem como a qualidade de vida, é necessário que o profissional esteja inserido não só no universo de seu trabalho mas também no mundo exterior que o beneficia interiormente, visto que este conjunto complementa-se para que o indivíduo permaneça em equilíbrio e obtenha a satisfação no seu cotidiano.

A aptidão de criar e manter um ambiente com presença reduzida de estressores organizacionais é uma exigência crescente, e todo empregador deve estar capacitado para gerir e reduzir o próprio estresse, bem como para auxiliar na diminuição das tensões de seus empregados.

\section{REFERÊNCIAS}

1. Genuíno SLV, Gomes MS, Moraes EM. O estresse ocupacional e a Síndrome de Burnout no ambiente de trabalho: suas influências no comportamento dos professores da rede privada do ensino médio de João Pessoa. Rev Anagrama. 2010;2:1-9.

2. Paschoal T, Tamayo A. Validação da escala de estresse no trabalho. Estud Psicol. 2004;9(1):45-52.

3. Acquadro Maran D, Varetto A, Zedda M, leraci V. Occupational stress, anxiety and coping strategies in police officers. Occup Med. 2015;65(6):466-73.

4. Carvalho AV, Serafim OCG. Administração de recursos humanos. São Paulo: Editora Pioneira; 2002.

5. Selye H. A syndrome produced by diverse nocuous agents. 1936. J Neuropsychiatry Clin Neurosci. 1998;10(2):230-1.

6. Lipp MEN. Manual do inventário de sintomas de stress para adultos de Lipp (ISSL). São Paulo: Casa do Psicólogo; 2000.

7. Tanure B, Carvalho Neto A, Santos CMM, Patrus R. Estresse, doença do tempo: um estudo sobre o uso do tempo pelos executivos brasileiros. Estud Pesq Psicol. 2014;14(1):65-88.

8. Rocha EL, Glima DMR. Distúrbios psíquicos relacionados ao trabalho. In: Ferreira Júnior M. Saúde no trabalho. Temas básicos para o profissional que cuida da saúde dos trabalhadores. São Paulo: Editora Roca; 2002. p.320-48.

9. Ulrich-Lai YM, Herman JP. Neural regulation of endocrine and autonomic stress responses. Nat Rev Neurosci. 2009;10(6):397-409.

10. CameloSHH, Angerami ELS. Sintomas de estresse em trabalhadores de cinco núcleos desaúde da família. Rev Latinoam Enferm. 2004;12(1):14-21.

11. Canova KR, Porto JB. O impacto dos valores organizacionais no estresse ocupacional: um estudo com professores de ensino médio. Rev Admin Mackenzie. 2010;11(5):4-31.

12. Lipp MEN. Stress: conceitos básicos. In: Lipp MEN. Pesquisa sobre estresse no Brasil: saúde, ocupações e grupos de risco. Campinas: Papirus; 1996. p.17-23.

13. Cotta RMM, Schott M, Azeredo CM, Franceschini SCC, Priore SE, Dias G. Organização do trabalho e perfil dos profissionais do Programa Saúde da Família: um desafio na reestruturação da atenção básica em saúde. Epidemiol Serv Saúde. 2006;15(3):7-18.

14. Gianasi LBS, Oliveira DC. A síndrome de burnout e suas representações entre profissionais de saúde. Estud Pesq Psicol. 2014;14(3):756-72.
15. Rezende R, Borges NMA, Frota OP.Síndrome de Burnoute absenteísmo em enfermeiros no contexto hospitalar: revisão integrativa da literatura brasileira. Com Ciênc Saúde. 2012; 23(3):243-52.

16. Batista JBV, Carlotto MS, Oliveira MN, Zaccara AAL, Barros EO Duarte MCS. Transtornos mentais em professores universitários: estudo em um serviço de perícia médica. J Res Fundam Care online. 2016;8(2):4538-48.

17. Schaufeli WB, Peeters MCW. Job stress and burnout among correctional officers: a literature review. Int J Stress Manag. 2000;7(1):19-48.

18. Maslach C, Schaufeli WB, Leiter MP. Job burnout. Annu Rev Psychol. 2001;52:397-422.

19. Vieira I. Conceito(s) de burnout: questões atuais da pesquisa e a contribuição da clínica. Rev Bras Saúde Ocup. 2010;35(122):269-76.

20. Coser A. Síndrome de Burnout: a doença laboral da contemporaneidade e a questão da responsabilidade civil. FADERGS. 2013;5(2):43-73.

21. Benevides-Pereira AMT. Burnout: quando o trabalho ameaça o bem-estar do trabalhador. São Paulo: Casa do Psicólogo; 2002.

22. Silva AA, Souza JM, Borges FN, Fischer FM. Health-related quality of life and working conditions among nursing providers. Rev Saúde Pública. 2010;44(4):718-25.

23. Gonçalves TB, Leitão AKR, Botelho BS, Marques RACC, Hosoume VSN, Neder PRB. Prevalência de síndrome de burnout em professores médicos de uma universidade pública em Belém do Pará. Rev Bras Med Trab. 2011;9(2):85-9.

24. Fabichak C, Silva-Junior JS, Morrone LC. Síndrome de burnout em médicos residentes e preditores organizacionais do trabalho. Rev Bras Med Trab. 2014;12(2):79-84.

25. Schermerhorn Jr JR, Hunt JG, Osborn RN. Fundamentos de comportamento organizacional. Porto Alegre: Editora Bookman; 1999.

26. Chiavenato I. Gestão de pessoas: o novo papel dos recursos humanos nas organizações. Rio de Janeiro: Elsever; 2004.

27. Merino E. Qualidade de vida no trabalho: conceitos básicos. Florianópolis: Editora Luz; 2000.

Endereço para correspondência: Claudia Eliza Papa do Prado - Rua 30 de Julho, 594, apto. 94 - CEP: 13465-500 - Americana (SP), Brasil -

E-mail: claudiapapa@live.com 